Research Article

\title{
Intelligent Informatization Early Warning Analysis of Agricultural Economy Based on Support Vector Sequential Regression Model
}

\author{
Ying Yang (iD) \\ School of Business Administration, Xinyang Agriculture and Forestry University, Xinyang, Henan 464000, China \\ Correspondence should be addressed to Ying Yang; 2018250002@xyafu.edu.cn
}

Received 21 October 2021; Accepted 15 November 2021; Published 30 November 2021

Academic Editor: Miaochao Chen

Copyright (C) 2021 Ying Yang. This is an open access article distributed under the Creative Commons Attribution License, which permits unrestricted use, distribution, and reproduction in any medium, provided the original work is properly cited.

\begin{abstract}
The development of science and technology has laid a solid foundation for the economic informatization of agriculture, and at the same time it brought technical guarantee for the development of agriculture, and the development of agriculture has provided an important material foundation for the development of science and technology. How to identify and deeply study agricultural economic informatization, give early warning to risk information, and ensure the steady development of the whole industry has become a key issue in the application of Internet technology in the field of agricultural development. This paper studies the present situation of agricultural economy informatization development process and applies support vector machine to forecast regional economic development level. The warning limit of agricultural economic growth rate is obtained on the basis of warning situation and warning indicator in early warning index system. The economic early warning model is established based on the support vector sequential regression method, and then the data is trained by MATLAB software to verify the rationality of the early warning model, and the accuracy and corresponding error of the model are given. Experimental results show that the prediction accuracy is $99.3 \%$, the error is less than 0.05 , and the prediction effect is relatively ideal, for agricultural economic intelligence information to provide accurate warning and agricultural economic research agricultural commercial development to provide support.
\end{abstract}

\section{Introduction}

In the development of information age, information management mode can promote the orderly development of all work. In the process of agricultural economic construction, sharing agricultural informatization technology through informatization management mode can effectively improve agricultural productivity to a certain extent, thus helping to accelerate the pace of overall economic construction. In the agricultural service system, it avoids the disadvantages of traditional management and reduces the difficulties of selling agricultural products, which not only has a good effect on the development of agriculture but also promotes the development of agricultural service system. Using the information of agricultural economic management, we should further broaden the marketing channel of agricultural products and improve the economic income of farmers to tide over the difficulties. In the process of agricultural production, by actively applying modern science and technology and advanced machinery and equipment, to the agricultural product processing renovation, to make the agricultural products in line with market demand, the applications of information technology to the agricultural economy management can improve the agricultural economic income and promote transformation of traditional agriculture towards knowledge-based agriculture and promote the sustainable development of agriculture [1]. Promoting the development of agricultural informatization plays an important role in the leapfrog development of agriculture and also promotes the scientific adjustment, optimization, and upgrading of agricultural structure. Promoting the informatization development of agricultural economy not only needs perfect infrastructure, but also has not fully realized the importance of informatization 
management in most rural areas. Economic growth and agriculture are inextricably linked. In order to better standardize the management of agricultural economy in our country, it is necessary to gradually introduce information technology, so as to effectively promote the development of agricultural economy information. The informatization development of agricultural economy is a long-term work, which needs the cooperation of many departments. The development of rural economy promotes farmers to change their ideas and promote informatization to permeate agricultural production and, at the same time, establish and perfect the scientific and reasonable agricultural development operation mechanism, so as to effectively enhance the ability and level of agricultural economic development. If we want to establish the corresponding agricultural economic information system, we need to comprehensively consider all aspects of agricultural development, comprehensively analyse and explore agricultural information resources, and gradually establish a comprehensive agricultural information management system. In addition, it is necessary to establish a timely service system and agricultural monitoring and early warning system according to the actual situation of rural areas in each region and market analysis.

Early warning belongs to the category of forecast; economic early warning belongs to the category of economic forecast. In general, in order to warn an object, it is necessary to collect and screen all kinds of information about the object, and put forward the key index system of warning index, including warning index and warning index, which can represent the characteristics of the object. The critical value of these indicators should also be determined according to practice and related theories, including the warning limit of the warning indicators and the police area of the warning indicators. With the critical value and data collected in real time, as well as the dynamic detection of these indicators, the warning target can be dynamically predicted by certain processing means or warning methods $[2,3]$. Economic early warning is to find the objective law of economic cycle and economic fluctuation through research and analysis and to detect the current situation of the current economy and future economic development, so as to ensure the stable development of the economy. Traditional early warning methods are often limited by expert experience and simple mathematical models, which make it difficult to achieve good results in dealing with high-dimensional features, small samples, and highly nonlinear models. As the algorithm has poor generalization ability, it has the disadvantages of slow learning speed and difficult convergence, which leads to unpredictability and cannot meet the objective requirements of macroeconomic early warning. Therefore, it is necessary to develop and improve the learning algorithm of economic early warning. A number of economic warnings have been proposed. Bayes classifier and neural network classifier are widely used in economic early warning. One of the key problems in the practical application of neural network is the construction of the network, which needs a large number of training samples, but the collection of these samples is very difficult in practical application. Traditional classification methods take the empirical risk minimization principle as the starting point and have poor generalization ability.

In the agricultural development of information technology, due to the popularity of the Internet, the price information of agricultural products is transparent development, agricultural products through the adaptation to the market environment. Support vector machine (SVM) is a machine learning algorithm developed based on statistical learning theory and structural risk minimization theory [4]. It uses support vector to determine the optimal partition hyperplane to segment data space and was first used for classification and recognition of complex information. At the same time, the hyperplane can be regarded as the greatest common divisor set of a class of data by adjusting the confidence interval range, and the hyperplane equation can be used as the regression model of the data. Because support vector machines can define a variety of kernel functions, nonlinear kernel functions such as radial basis kernel function (RBF) can map samples to high-dimensional space operations, which makes support vector machines have the ability to deal with nonlinear data. Support vector machine (SVM) is a powerful learning tool induced by statistical learning theory [5]. Especially in the small sample, high-dimensional, nonlinear data space, support vector machine can make full use of the information provided by various features; it has good generalization ability in the learning process. Support vector machine training is suitable for short-term prediction with less data and less sample demand and modelling workload.

\section{Related Work}

In order to improve the level of agricultural economic management information, the establishment of a perfect information platform can improve the sharing degree of information resources and improve the utilization rate of agricultural information. The rapid development of agricultural information technology can only be promoted after ensuring that agricultural information management is really implemented in every link. Economists put forward a new topic-measurement and prediction of economic fluctuations. Jin et al. elaborated the theoretical basis, basic principles, and methods of agricultural early warning and the general process of agricultural system early warning [6]. In accordance with the four stages of the early warning logic process, Roy and Chakraborty focus on two links: clear warning situation and analysis of warning signs, qualitatively analyses of the grain warning situation index and grain predictor index, and providing a basic framework for the conception of the grain prediction index system [7]. Melki et al. focus on the establishment of a food early warning system and discuss the characteristics of the food early warning system, the technical methods for establishing the food early warning system, and the content and procedure of the food early warning system from the study of the situation and characteristics of the grain yield fluctuation [8]. Tao et al. analysed the food alarm situation through systematic methods using five indicators, namely, historical change characteristics of grain production, average growth rate of world grain output in the same period, annual 
population growth rate, maximum and minimum net grain export rates, and necessary grain reserves, etc. [9]. Zhou et al. systematically elaborated the theoretical basis of food security, food security, and early warning system and proposed the choice of four alternative modes to guarantee agricultural security, as well as the food security early warning indicator system and the sensitive range of alarm indicators [10].

Economic early warning is for statistician to analyse macroeconomic prosperity of the national economy; economic sentiment analysis includes two aspects, one is economic monitoring; the former is mainly used to determine the boom strength now and change direction, which is used to forecast the future status of economic operation of the point, predicting the future of economic strength, so often calling economic boom analysis economic monitoring early warning. The Bayesian classifier assumes that the probability density function of early warning conforms to the independent distribution of Gaussian, which has the advantages of no iterative operation and less computation. However, there are two disadvantages in the practical application of Bayesian classifier: first, if the probability density function of the early warning does not conform to the Gaussian distribution, it will not get good classification results; second, the classifier trained with a small number of training sample sets will produce errors when classifying a large number of sample spaces [11]. Wang et al. proposed the economic early warning based on Bayesian decision classification [4]. This early warning system based on probabilistic pattern classification has opened a new research field for economic early warning methods. The basic idea of neural network classifier is to train ANN with training sample set to determine the connections and weights between nodes. Each node in the input layer corresponds to each attribute of economic early warning, and the result of the output layer is the number of categories of early warning degree. Karimipour et al. used the multilayer network and trained the network with the backpropagation method [12]. Liang et al. used genetic algorithm and support vector combination model to modify the autoregressive model, and the empirical analysis results showed that the preaccuracy of stock index series of the model was controlled within 7\% [13]. Panahi et al. introduced the least squares support vector machine into water quality prediction and predicted the high-intensity acid index of river waters for two consecutive years, achieving higher prediction accuracy than the neural network model [14].

This paper combines support vector sequential regression with macroeconomic early warning research, tries to establish macroeconomic early warning method system based on support vector sequential regression, and extends the theory and method of support vector machine. At the same time, it is combined with the actual data of economic early warning empirical analysis, in order to achieve the combination of theory and practice.

\section{Support Vector Sequential Regression Model}

3.1. Support Vector Data Describes Early Warning Techniques. Support vector data description early warning technology divides early warning work into two steps; the early warning steps are shown in Figure 1.
Support vector data is distinguished from normal data and other data, and a class classifier based on support vector data description is established [15], which includes all normal data objects that can be confirmed in a hypersphere to distinguish it from other data outside the hypersphere. Support vector data identifies abnormal data in other data outside the hypersphere. Other data outside the sphere will be judged by economic warning experts as abnormal data, and the platform will issue economic abnormal warning.

3.2. Support Vector Data Description. Vapnik's statistical learning theory argues [16] that if the data volume of a sample is relatively small, it is more effective to classify a sample by directly searching for a closed region than by estimating its probability density. Such a class of directly searching for a closed region is known as data description.

For a sample set containing $N$ "normal" data objects:

$$
\left\{x \mid x_{i}, i=1, \cdots, n\right\} \text {. }
$$

The resulting hypersphere is described by the center point $A$ and the radius $R$. To form a compact sphere boundary, the optimization problem of data description can be expressed as

$$
\begin{gathered}
\min L(R)=R^{2}, \\
R^{2}-\left(x_{i}+a\right)\left(x_{i}-a\right)^{T} \geq 0 .
\end{gathered}
$$

According to formulas (2) and (3), the following Lagrange function is defined:

$$
L(R, a, \wedge)=R^{2}-\sup \left\{\lim _{n \longrightarrow \infty} \sum_{i=1}^{n} a_{i}\left[R^{2}-\left(x_{i}+a\right)\left(x_{i}-a\right)^{T}\right]\right\} .
$$

Lagrange coefficient $\alpha_{i} \in \Lambda$, and $\alpha_{i} \geq 0$. Take the partial derivative of formula (4) with respect to $R$ and make it equal to 0 , and get

$$
\sum_{i=1}^{n} a_{i}=1
$$

Take the partial derivative of formula (4) with respect to $A$ and make it equal to 0 , and combine formula (5) to obtain

$$
\sum_{i=1}^{n} a_{i} x_{i}=b
$$

Substitute formulas (5) and (6) into formula (4) to obtain the optimization equation:

$$
\max L=\sup \left\{\lim _{n \longrightarrow \infty} \sum_{i=1}^{n} a_{i}\left(x_{i} \cdot x_{i}\right)+\lim _{n \longrightarrow \infty} \sum_{i=1}^{n} \sum_{i=1}^{n} a_{i} a_{j}\left(x_{i} \cdot x_{j}\right)\right\},
$$

$$
\sum_{i=1}^{n} a_{i}=1, \quad a_{i} \geq 0
$$

According to the KKT condition, most $\alpha_{i}=0$ and a few $\alpha_{i} \geq 0$. The bounds of the hypersphere are determined by the 


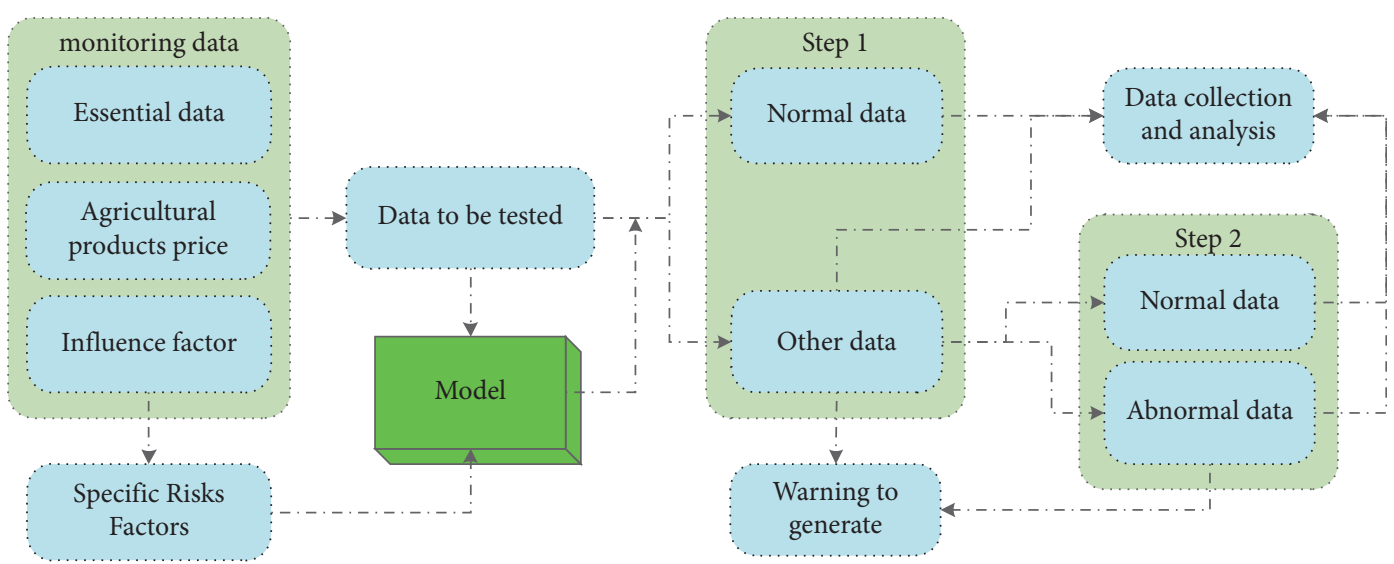

FIGURE 1: Support vector data describes the warning steps.

sample $x_{i}$ corresponding to nonzero $\alpha_{i}$, which is called the support vector $(\mathrm{SV})$. For the given $\wedge$, the spherical center $r$ can be calculated by formula (6), and an optional support vector can be calculated by the following formula:

$$
R^{2}-\left(x_{i}+a\right)\left(x_{i}-a\right)=0 .
$$

For the data $z$ to be detected, let

$$
f(z)=(z+r)(z-a)^{T} .
$$

Formula (10) can also be transformed into

$$
f(z)=(z \cdot z)+2 \sum_{i=1}^{n} a_{i}\left(z \cdot x_{i}\right)-\sum_{i=1}^{n} \sum_{i=1}^{n} a_{i} a_{j}\left(x_{i} \cdot x_{j}\right) .
$$

Consider that, in formula (11), for $z$ to be tested,

$$
\sum_{i=1}^{n} \sum_{i=1}^{n} a_{i} a_{j}\left(x_{i} \cdot x_{j}\right)=\text { const. }
$$

Let

$$
\begin{aligned}
& g(z)=(z \cdot z)+2 \sum_{i=1}^{n} a_{i}\left(z \cdot x_{i}\right), \\
& \lambda(z)=\frac{g(z)}{g\left(x_{e}\right)} .
\end{aligned}
$$

$X_{e}$ is any support vector. Therefore, whether $Z$ is outside the hypersphere can be determined according to the following formula :

$$
\lambda(z)= \begin{cases}>1, & z \text { is outside the hypersphere } \\ \leq 1, & z \text { is in the hypersphere. }\end{cases}
$$

It can be seen that formulas (13) and (14) are used to determine the state of the point $Z$ to be determined, which saves the calculation process of radius $R$ and center point $R$ and simplifies the calculation. However, as the boundary shape determined by the optimization equation (7) is single, and the space formed by the boundary region is too large and not compact enough, it is easy to bring abnormal data into the scope of the hypersphere. The kernel method (KM) is used to map data objects in the input space to the kernel space to solve this problem. Replace the inner product operation with the kernel function that meets Mercer's conditions; then the optimization equation (7) of the attribute space is transformed into

$$
\max L=\sup \left\{\lim _{n \longrightarrow \infty} \sum_{i=1}^{n} a_{i} K\left(x_{i} \cdot x_{i}\right)+\sum_{i=1}^{n} \sum_{i=1}^{n} a_{i} a_{j} K\left(x_{i} \cdot x_{j}\right)\right\} .
$$

The constraint conditions remain unchanged, and formula (11) becomes

$$
f(z)=K(z \cdot z)+2 \lim _{n \longrightarrow \infty} \sum_{i=1}^{n} a_{i} K\left(z \cdot x_{i}\right)-\lim _{n \longrightarrow \infty} \sum_{i=1}^{n} \sum_{i=1}^{n} a_{i} a_{j} K\left(x_{i} \cdot x_{j}\right) .
$$

Formula (13) becomes

$$
g(z)=K(z \cdot z)+2 \lim _{n \longrightarrow \infty} \sum_{i=1}^{n} a_{i} K\left(z \cdot x_{i}\right)
$$

This kind of classification method based on kernel method that directly seeks closed region is called support vector data description [17]. In particular, Gaussian radial basis kernel functions are selected.

$$
K(x, y)=\exp \left(\frac{-\|x+y\|^{2}}{\sigma^{2}}\right)
$$

Sigma can be given in advance. Given different $\sigma$ it is possible to produce different hyperspherical boundaries for the same sample set. After introducing Gaussian radial basis kernel function, formulas (16) and (18) can be simplified as

$$
\begin{aligned}
& \max L=\sup \left\{1-\lim _{n \longrightarrow \infty} \sum_{i=1}^{n} \sum_{i=1}^{n} a_{i} a_{j} K\left(x_{i} \cdot x_{j}\right)\right\}, \\
& g(z)=1-2 \lim _{n \longrightarrow \infty} \sum_{i=1}^{n} a_{i} K\left(z \cdot x_{i}\right) .
\end{aligned}
$$

After introducing the kernel method to form the boundary of the normal data set, if the data object $z>1, z$ is suspected abnormal data. 
3.3. Support Vector Sequential Regression Model Early Warning Process. Based on the study of the change characteristics of agricultural economy, the evolution process still has obvious autocorrelation and linear characteristics, so the model is set as support vector sequential regression model to make linear prediction first and then to characterize the missing nonlinear characteristics [18]. The specific prediction process is shown in Figure 2.

The specific prediction steps are as follows: after the original data is preprocessed, the sample data is divided into training set and test set according to the idea of cross validation. Then, the training set model was used to build the model according to the modelling process of the model. After the smooth processing, autoregression, moving average grading, and BIC criterion judgment, the model was established and the sequence was predicted, and the firststage prediction sequence was obtained. The residual predicted by ARIMA model is calculated by referring to the test set data, and the formula is

$$
e_{t}=y_{t}-y_{t}^{\prime} .
$$

In the above formula, $y_{t}$ is the real data of the test set. After obtaining the residual sequence, it is necessary to conduct white noise test on the residual data. If the residual sequence is not pure random white noise, it indicates that the residual sequence contains useful information and the prediction of the sequence by the model is not sufficient. The prediction sequence of the model is taken as the input term, and the corresponding residual sequence is taken as the output term to reduce the influence caused by the difference in numerical size and improve the convergence speed of the model and the accuracy of the final prediction. The normalized processing of the input quantity is required, and the transformed formula is as follows:

$$
\widehat{x}=\left(N_{\max }-N_{\min }\right)\left(\frac{x-x_{\min }}{x_{\max }-x_{\min }}\right) .
$$

In the above formula, $N_{\max }$ and $N_{\min }$ are the maximum and minimum values of the target interval, set as $[-1,1] ; x_{\max }$ and $x_{\min }$ are the maximum and minimum values of the data to be normalized; $x$ is the original value. The normalized data were obtained and then brought into the model for training. In the process, the penalty coefficient $C$ and the kernel parameter $\gamma$ were optimized by PSO algorithm. After the training, residual can be predicted, and the residual prediction sequence can be obtained by inverse normalization of the predicted values. So far, the initial predicted value of ARIMA model and residual predicted value of SVR model have been obtained. The final work is to fuse the results of the two parts. The specific operation is to directly sum the results of the two parts, and the formula is as follows:

$$
y_{t}^{\prime \prime}=y_{t}^{\prime}+e_{t}^{\prime} .
$$

3.4. Conceptual Design of Data Warehouse. Data warehouse is the core of agricultural economic information system and the basis of information analysis and data mining. The conceptual model design of data warehouse must be based on the actual investigation; combined with the actual information analysis needs and the structure of the data source, the design of the wrong data warehouse model will lead to the failure of the whole intelligent information system and produce wrong information. The conceptual design of data warehouse model mainly involves the design of dimension table and fact table and the relationship between dimension table and fact table [19]. Star model and snowflake model are often used in data warehouse design. However, star models should be used wherever possible because the dimension tables are intended to provide a convenient perspective for analysis. While the snowflake model can reduce dimensional table spaces, it increases the difficulty of analysing data through dimensions and complicates data warehouse structures. In the design of data warehouse, only the snowflake structure is adopted in the design of product dimension. This is because the product dimension contains category attributes, and data inconsistency is more likely to occur through attributes described by characters. Therefore, the snowflake structure is adopted to avoid this situation. The overall data warehouse model is shown in Figure 3.

With the granularity of "economy," geographic dimension records the geographic information associated with the price of agricultural products, which is one of the key dimensions of data warehouse and the most commonly used dimension in data analysis, as well as the dimension most associated with other fact tables and dimension tables [20]. Its structure is proxy primary key, candidate key, and locale name. Geography Key is the primary key of the dimension table, and Geography Alternate Key is the candidate key. This field can be used to trace back to the data source, increasing the reliability of the data warehouse. In the design of this latitude, the actual situation of agricultural economy was taken into account, and the hierarchical structure from region to county and city to market was designed, instead of applying general geographical information directly; otherwise it could not meet the needs of practical analysis.

The time dimension is the most commonly used dimension in data analysis. Its structure is proxy primary key, year, month, day, festival, and special event. Among them, the Chinese name attributes of year and month are set to facilitate the display of the later analysis, and the properties of Day of Year and Day of Month are set to facilitate the comparison and calculation of the price of agricultural products in the same period. Because business topics are time series. The time dimension of agricultural economic data warehouse records the time description information with the granularity of "day." When designing the time dimension, we must consider the needs of data analysis and data mining, rather than simply extracting data from the system [21, 22]. For example, considering that the fluctuation of agricultural product price is closely related to holidays, the attribute of "holiday indication" should be added in the design of time dimension. Also considering the influence of special factors on the price of agricultural products special event attributes should be set. Finally, "solar terms" and "seasons" should also be added which have a greater relationship with the price of agricultural products. 


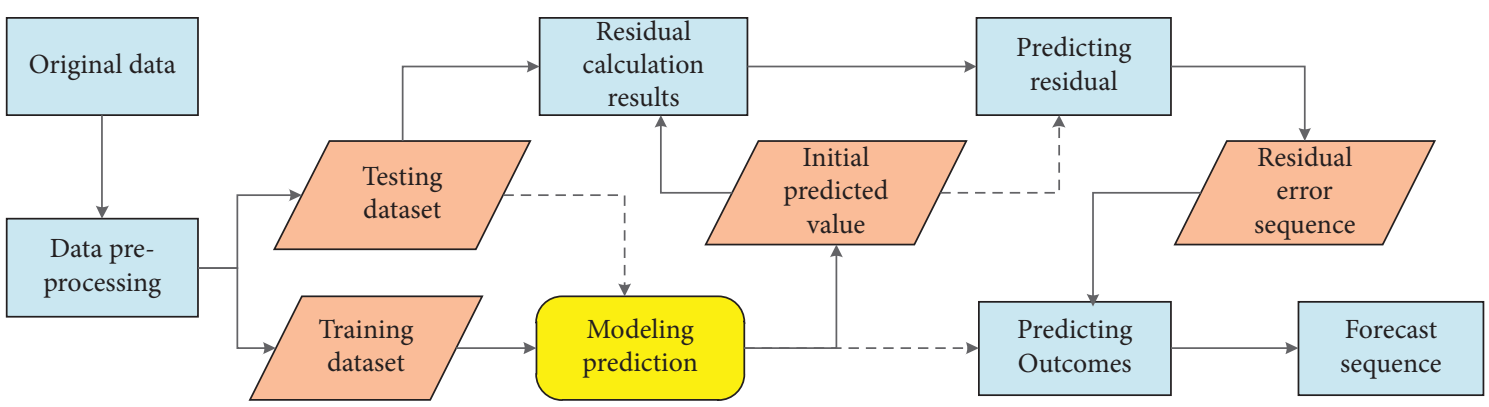

FIGURE 2: Support vector sequential regression model early warning flowchart.

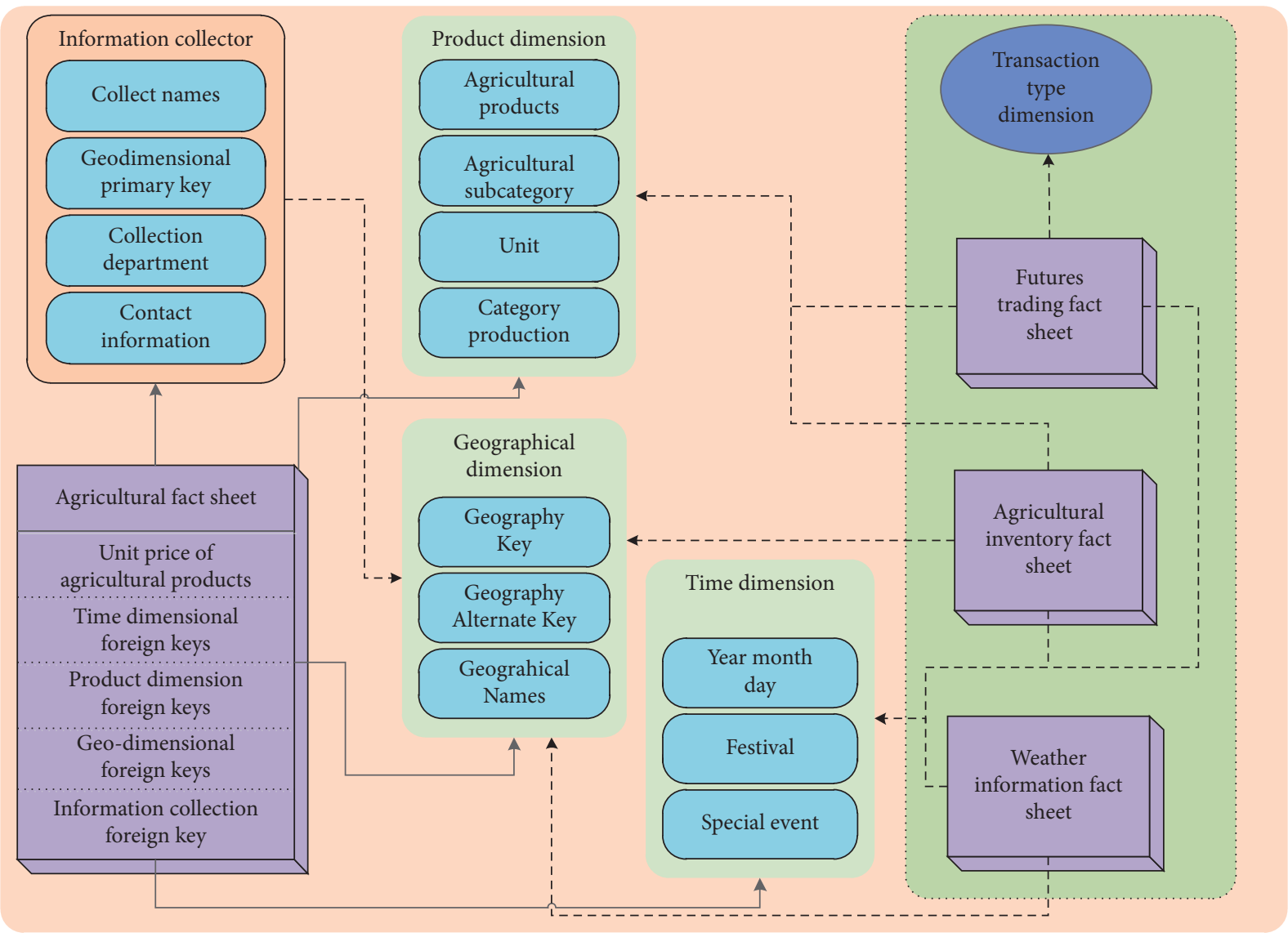

FIgURE 3: Data warehouse overall model diagram.

The product dimension records the agricultural product information with the granularity of "agricultural product," and its structure is as follows: agricultural product name, agricultural subcategory, unit, and category production. The product latitude of agricultural products is a complex design that depends primarily on the end user's analytical perspective. But it must also consider the interface with other data sources; special design is not conducive to the unification of data sources and the expansion of data warehouse $[23,24]$. The product dimension table is the dimension table that changes most frequently of all dimension tables. It changes over time. It is a gradual dimension. Instead of updating the original data when loading data, a new row is added to preserve the historical data. Start Date and End Date are combined to indicate whether the current data row is invalid. The information provider dimension mainly records the relevant information of information providers in each market, which is linked to the geographical dimension. It is mainly used to support the analysis of the contribution degree of information provision and the calculation of performance appraisal.

\section{Realization and Analysis of Intelligent Informatization Early Warning of Agricultural Economy}

4.1. Realization of Economic Early Warning. The research method of economic warning situation index is mainly to determine the warning limit according to various parallel objective principles or standards and then to reasonably 
synthesize all kinds of results, so as to get scientific conclusions and determine the economic warning degree. These principles mainly include majority principle, half principle and median principle, and average principle. This thesis mainly adopts the majority principle. Based on the warning situation and warning indicators in the early warning indicator system, combined with the actual data, with the grain planting area $\left(X_{1}\right)$, agricultural output $\left(X_{2}\right)$, the planting area $\left(X_{3}\right)$, production $\left(X_{4}\right)$, the affected area $\left(X_{5}\right)$, agricultural output $\left(X_{6}\right)$, irrigation area $\left(X_{7}\right)$, find $\left(X_{8}\right)$, the total power of agricultural machinery $\left(X_{9}\right)$, agricultural labour productivity $\left(X_{10}\right)$, and other warning signs with the weight of the index as the input vector $x$, the output variable $y=\{1,2,3,4,5\}$ is a multiclass ordered set, where 1 represents no alarm, 2 represents light alarm, 3 represents medium alarm, 4 represents serious alarm, and 5 represents huge alarm.

For quite a long time in the past, the overall economic situation of most of the cases of economic early warning alert is in warning state; on the basis of the qualitative knowledge and the economy early warning index of time series data, from small to large order again, down from a minimum value, the data interval accounting for $2 / 3$ of the total number was selected as the safety alarm limit, i.e., no alarm limit. The remaining $1 / 3$ data interval was divided into light alarm limit, medium alarm limit, heavy alarm limit, and giant alarm limit. The division of light alarm limit, medium alarm limit, heavy alarm limit, and giant alarm limit could be carried out according to equal distance. Thus, the warning limit of agricultural economic growth rate is shown in Figure 4.

On the basis of inheriting the traditional early warning system, modern early warning system makes full use of the research results of support vector system theory, avoids or minimizes the human factors in the early warning process, and makes the early warning process more objective and reliable.

4.2. Model Test Accuracy Analysis. In order to enhance the applicability of the model and improve the accuracy, the following processing is done in data entry in this paper: the original sample is divided into training set and test set according to the ratio of $7: 3$ which is most commonly used for small samples, and $70 \%$ samples are randomly extracted to form the training set, and the remaining $30 \%$ are automatically grouped into the test set of the same group. The specific random process is to use the sample random function in the random module of Python language program to generate random numbers in each category and then select samples with corresponding serial numbers to form test sets. Due to the randomness of training set and test set, in order to reduce the error caused by randomness, the usual input of one data set is changed to input multiple data sets. In order to make the final prediction accuracy convergent and the model not too complex, the process of randomly dividing the training set and test set was repeated for 10 times to obtain 10 groups of different data sets. Ten groups of data sets were input into the model using the cyclic algorithm, and the decision coefficients of each group of classification results were obtained to observe the classification

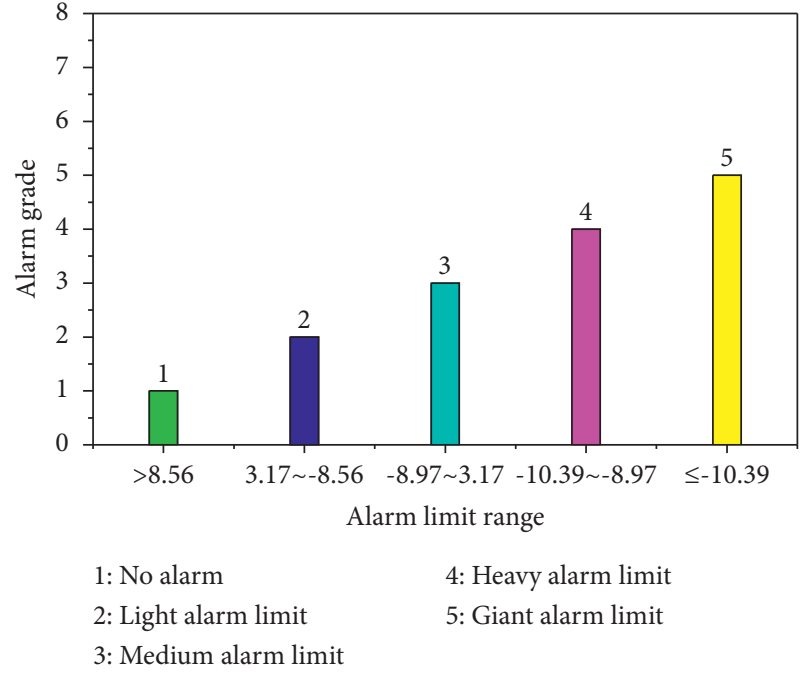

FIGURE 4: Warning limit chart of agricultural economic growth rate.

accuracy. The cycle was repeated 10 times, and the average value was calculated by integrating the decision coefficients of 10 groups to obtain the decision coefficients of the model, so as to observe the effect of early warning. For the highest prediction accuracy in the test model, observe the influence on the model accuracy when the number of iterations is 50 , 100,500 , and 1000, respectively, and the obtained decision coefficients are shown in Figure 5.

From the above test results, the model support vector machine can be obtained, and the prediction accuracy is the highest. Firstly, the influence of different iterations on the prediction accuracy of the model in support vector machine was observed. As the number of iterations increases, the prediction accuracy increases gradually. When the number of iterations is 1000 , the prediction accuracy reaches $99.3 \%$, and the prediction effect is relatively ideal.

4.3. Prediction Error Test Analysis. According to the interval into which the values calculated by the early warning model fall, interpolation test is performed on the training sample data. When the risk prediction model is trained by MATLAB software, error statistics of simulation results are performed, as shown in Figure 6.

After many times of training, the figure gives the training curve of different indexes with the minimum error of the initialized data in MATLAB software. The model error diagram with the error less than 0.05 obtained after repeated training and learning shows that the network learning is successful within a limited range. After successful training, the remaining sample data are input into the trained model for testing, and the output result is between 0 and 1 . The closer the result is to 1 , the higher the credit degree is.

4.4. Implementation of KPIs Functionality. In the final facility stage of the initial utilization of agricultural economic intelligent information system, the corresponding ETL 


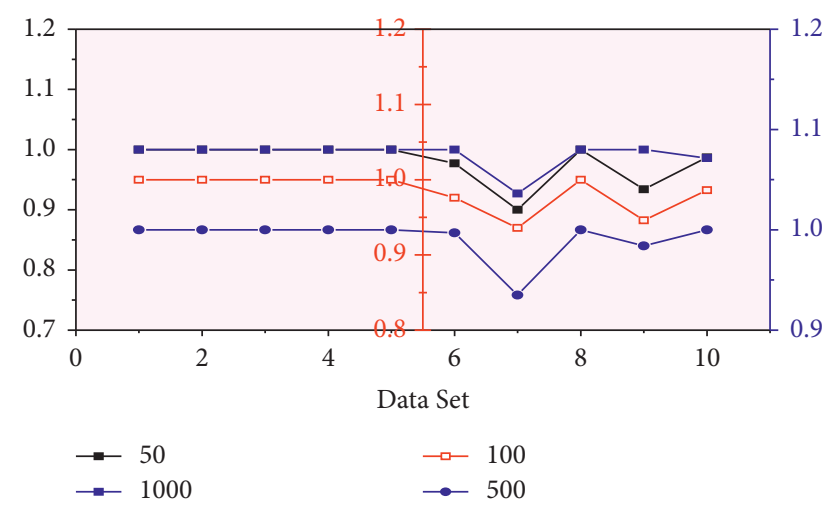

FIgURE 5: Model accuracy determination coefficient diagram.

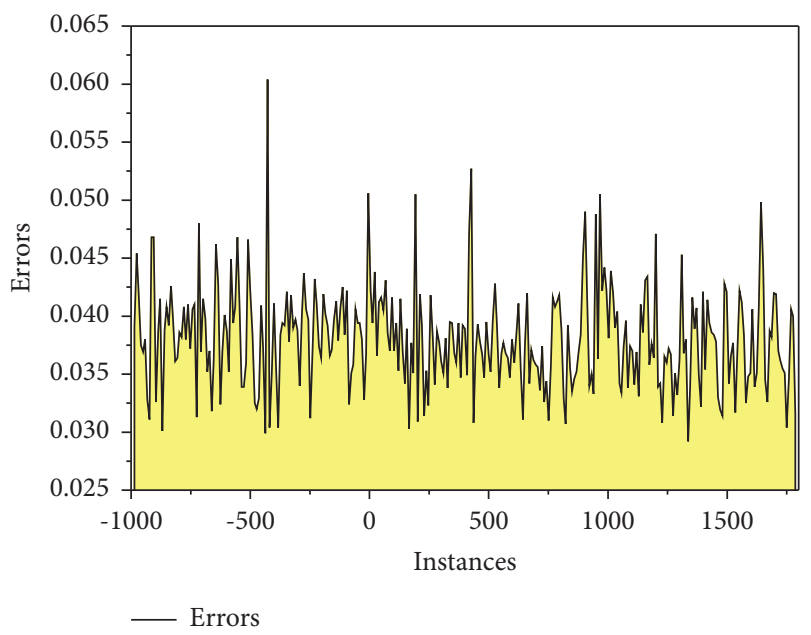

FIgURE 6: Error statistics of simulation results.

program is designed based on the data warehouse model, relevant data are imported, multidimensional data are cut into blocks by combining geographical latitude and time dimension, and detailed information is obtained by drilling down the data, which is displayed synchronously through charts. In the end, the distribution of agricultural and sideline products warning in the corresponding time period is shown in Figure 7.

As can be seen from the figure, agricultural economy continues to improve, planting structure continues to optimize, pig production capacity continues to recover, agricultural investment grows rapidly, agricultural trade continues to improve, and farmers' income steadily increases. Poultry meat, aquatic products, pork, cattle, and sheep are in moderate alert state, the rest are in no alert state, and the economy continues to grow. Agricultural economy monitors early warning result to show, agricultural economy picks up, and agricultural economy early warning index is flat. Agricultural and economic indicators have picked up to varying degrees, and agricultural labour productivity has continued to improve. This will lay a solid foundation for stabilizing market expectations and ensuring food security. It is expected that the rise in agricultural prices will continue to fall. On the whole, the agricultural economy is expected to continue to recover. In

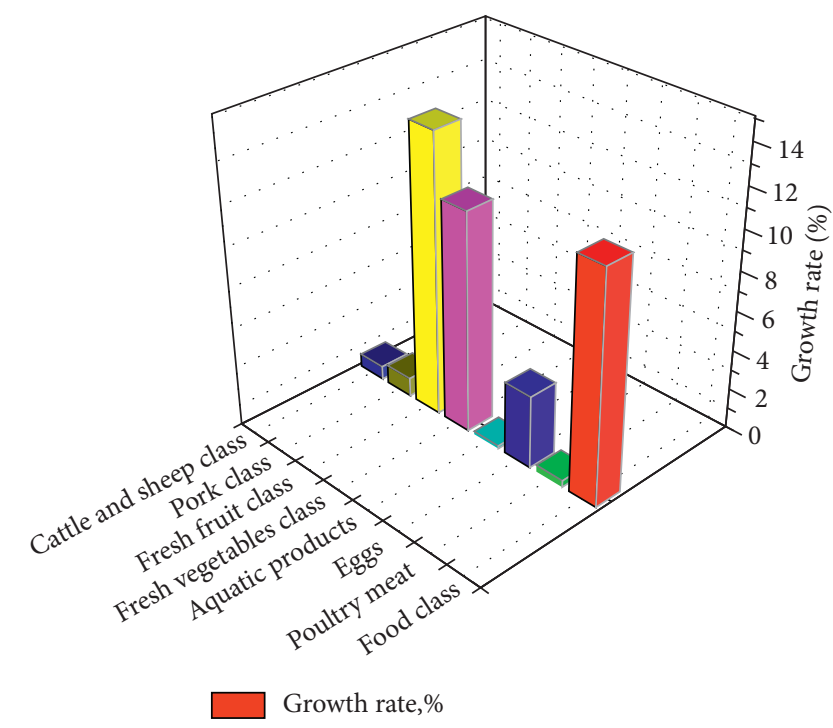

FIgURE 7: Warning distribution map of agricultural and side-line products.

the first quarter of next year, due to the impact of the epidemic caused by a low year-on-year base, the economic index will significantly recover.

\section{Conclusion}

In the development of information era, informatization management mode can promote the orderly conduct of the work, so in the process of agricultural economic development, the informatization management mode can promote the comprehensive construction of agricultural economy information, due to the comprehensiveness of agricultural technology to achieve the promotion, so in the process of information management, agricultural information technology to share, to a certain extent, can effectively improve agricultural productivity, thus helping to accelerate the pace of overall economic construction. On the basis of statistical learning theory and support vector machine theory, this paper combines support vector sequential regression algorithm with macroeconomic early warning research and establishes an economic early warning model based on support vector sequential regression. Through the preparation of MATLAB software program, combined with the actual economic data, economic early warning prediction warning ranges, using empirical analysis method of economic early warning system construction research. Analysis of agricultural economic development situation, with early warning index system of early warning and warning sign index as the basis, combined with the actual data, chose to have important effects on agricultural output of the nine factors' index; using support vector machine (SVM) regression machine order for inspection system and prediction, agricultural economy early warning model is established, putting forward building the early warning system of the specific steps and running the process. The prediction results and actual error analysis are obtained. Agricultural early warning system is a dynamic optimization 
process with many variables, and the determination of warning limit should change with the change of conditions. The short-term warning and medium-long-term warning can achieve better warning effect. Although the economic early warning effect is good, due to the uncertainty and fuzziness of the economic system, it is necessary to further improve the model and adjust and optimize the early warning index parameters in the next step.

\section{Data Availability}

The data used to support the findings of this study are available from the corresponding author upon request.

\section{Conflicts of Interest}

The author declares no conflicts of interest or personal relationships that could have appeared to influence the work reported in this paper.

\section{References}

[1] J. H. Britt, R. A. Cushman, C. D. Dechow et al., "Invited review: learning from the future-a vision for dairy farms and cows in 2067," Journal of Dairy Science, vol. 101, no. 5, pp. 3722-3741, 2018.

[2] T. Hess, J. Knox, I. Holman, and C. Sutcliffe, "Resilience of primary food production to a changing climate: on-farm responses to water-related risks," Water, vol. 12, no. 8, p. 2155, 2020.

[3] S. K. Wegren, A. M. Nikulin, and I. Trotsuk, "Russian agriculture during Putin's fourth term: a SWOT analysis," Postcommunist Economies, vol. 31, no. 4, pp. 419-450, 2019.

[4] X.-J. Wang, G.-T. Zeng, K.-X. Zhang, H.-B. Chu, and Z.-S. Chen, "Urban real estate market early warning based on support vector machine: a case study of Beijing," International Journal of Computational Intelligence Systems, vol. 13, no. 1, pp. 153-166, 2020.

[5] C. Jiang, Y. Fang, P. Zhao, and J. Panneerselvam, "Intelligent UAV identity authentication and safety supervision based on behavior modeling and prediction," IEEE Transactions on Industrial Informatics, vol. 16, no. 10, pp. 6652-6662, 2020.

[6] X. Jin, X. Shi, J. Gao, T. Xu, and K. Yin, "Evaluation of loss due to storm surge disasters in China based on econometric model groups," International Journal of Environmental Research and Public Health, vol. 15, no. 4, p. 604, 2018.

[7] A. Roy and S. Chakraborty, "Support vector regression based metamodel by sequential adaptive sampling for reliability analysis of structures," Reliability Engineering \& System Safety, vol. 200, Article ID 106948, 2020.

[8] G. Melki, A. Cano, V. Kecman, and S. Ventura, "Multi-target support vector regression via correlation regressor chains," Information Sciences, vol. 415-416, pp. 53-69, 2017.

[9] T. Tao, E. Zio, and W. Zhao, "A novel support vector regression method for online reliability prediction under multistate varying operating conditions," Reliability Engineering \& System Safety, vol. 177, pp. 35-49, 2018.

[10] Q. Zhou, X. Shao, P. Jiang, H. Zhou, and L. Shu, “An adaptive global variable fidelity metamodeling strategy using a support vector regression based scaling function," Simulation Modelling Practice and Theory, vol. 59, pp. 18-35, 2015.

[11] X. Zhou and W. Chen, "The impact of informatization on the relationship between the tourism industry and regional economic development," Sustainability, vol. 13, no. 16, p. 9399, 2021.

[12] A. Karimipour, S. A. Bagherzadeh, A. Taghipour, A. Abdollahi, and M. R. Safaei, "A novel nonlinear regression model of SVR as a substitute for ANN to predict conductivity of MWCNT-CuO/water hybrid nanofluid based on empirical data," Physica A: Statistical Mechanics and Its Applications, vol. 521, pp. 89-97, 2019.

[13] H. Liang, J. Zou, Z. Li, M. J. Khan, and Y. Lu, "Dynamic evaluation of drilling leakage risk based on fuzzy theory and PSO-SVR algorithm," Future Generation Computer Systems, vol. 95, no. 5, pp. 454-466, 2019.

[14] M. Panahi, A. Gayen, H. R. Pourghasemi, F. Rezaie, and S. Lee, "Spatial prediction of landslide susceptibility using hybrid support vector regression (SVR) and the adaptive neuro-fuzzy inference system (ANFIS) with various metaheuristic algorithms," The Science of the Total Environment, vol. 741, Article ID 139937, 2020.

[15] M. Panahi, N. Sadhasivam, H. R. Pourghasemi, F. Rezaie, and S. Lee, "Spatial prediction of groundwater potential mapping based on convolutional neural network $(\mathrm{CNN})$ and support vector regression (SVR)," Journal of Hydrology, vol. 588, Article ID 125033, 2020.

[16] H. S. Dhiman, D. Deb, and J. M. Guerrero, "Hybrid machine intelligent SVR variants for wind forecasting and ramp events," Renewable and Sustainable Energy Reviews, vol. 108, pp. 369-379, 2019.

[17] V. L. Shabanov, M. Y. Vasilchenko, and E. A. Derunova, "formation of an export-oriented agricultural economy and regional open innovations," Journal of Open Innovation: Technology, Market, and Complexity, vol. 18, no. 7, p. 32, 2021.

[18] Y. Tong, "Model for evaluating the green supply chain performance under low-carbon agricultural economy environment with 2-tuple linguistic information," Journal of Intelligent and Fuzzy Systems, vol. 32, no. 3, pp. 2717-2723, 2017.

[19] A. M. U. Khurramov, "The role and role of digital economy and information technology in the agricultural sector," International Journal on Integrated Education, vol. 3, no. 2, pp. 42-44, 2020.

[20] M. Zhao, Z. Chen, H. Zhang, and J. Xue, "Impact assessment of growth drag and its contribution factors: evidence from China's agricultural economy," Sustainability, vol. 10, no. 9, p. 3262, 2018.

[21] A. Onan, S. Korukoğlu, and H. Bulut, "Ensemble of keyword extraction methods and classifiers in text classification," Expert Systems with Applications, vol. 57, pp. 232-247, 2016.

[22] H. Azarbonyad, M. Dehghani, M. Marx, and J. Kamps, "Learning to rank for multi-label text classification: combining different sources of information," Natural Language Engineering, vol. 27, no. 1, pp. 1-23, 2020.

[23] A. Onan, "An ensemble scheme based on language function analysis and feature engineering for text genre classification," Journal of Information Science, vol. 44, no. 1, pp. 28-47, 2018.

[24] S. Xu and Y. Xiang, "Frog-GNN: multi-perspective aggregation based graph neural network for few-shot text classification," Expert Systems with Applications, vol. 176, Article ID $114795,2021$. 